\title{
Mandenkan
}

MANDENIKAN Bulletin semestriel d'études linguistiques mandé

$58 \mid 2017$

Numéro 58

\section{Morphologie verbale de la langue goo}

Verbal morphology of the Goo language

ГЛАГОЛЬНАЯ МОРФОЛОГИЯ

ЯЗЫКА

ГОО

\section{Ekaterina Aplonova}

\section{(2) OpenEdition}

Journals

\section{Electronic version}

URL: https://journals.openedition.org/mandenkan/1207

DOI: 10.4000/mandenkan.1207

ISSN: 2104-371X

\section{Publisher}

Llacan UMR 8135 CNRS/Inalco

\section{Printed version}

Date of publication: 1 December 2017

ISSN: 0752-5443

\section{Electronic reference}

Ekaterina Aplonova, "Morphologie verbale de la langue goo", Mandenkan [Online], 58 | 2017, Online since 05 March 2018, connection on 08 July 2021. URL: http://journals.openedition.org/mandenkan/ 1207 ; DOI: https://doi.org/10.4000/mandenkan.1207

This text was automatically generated on 8 July 2021.

\section{(c) (i) 8 (2)}

Les contenus de Mandenkan sont mis à disposition selon les termes de la Licence Creative Commons Attribution - Pas d'Utilisation Commerciale - Partage dans les Mêmes Conditions 4.0 International. 


\title{
Morphologie verbale de la langue
}

\section{goo}

\author{
Verbal morphology of the Goo language
}

ГЛАГОЛЬНАЯ МОРФОЛОГИЯ

ГОО

Ekaterina Aplonova

\section{Introduction}

1 La langue goo est parlée dans la région des Dix-Huit Montagnes à l'Ouest de la Côte d'Ivoire. Le goo est une des langues du groupe linguistique mandé-sud. Les langues les plus proches du goo sont le dan et le toura. Le nombre des Goo en Côte-d'Ivoire est d'environ 7 000. Jusqu'à 2012 le goo était occasionnellement mentionné comme « un parler mixte dan-toura » (Bearth 1971:5). Son statut en tant qu'une langue à part a été établi en 2012. Selon la lexicostatistique, la distance entre le goo et le toura correspond à une existence séparée depuis environ 1300 ans $^{1}$, et la distance entre le goo et le dangoue ta est de 160 ans (Vydrin 2013 : 189).

2 Pour le moment, il n'existe aucune publication en goo sauf un petit guide d'orthographe où est incluse un conte en goo Ge ièdääbäyilit ('L'arbre rouge'). Il n'y a pas d'émissions radio ${ }^{2}$, ni enseignement scolaire. Au cours de ces dernières années, le goo a été l'objet de plusieurs publications. Deux articles de Valentin Vydrin ont été publiés $(2013,2014)$ où des données préliminaires sur la langue goo ont été présentées : une description du système phonologique, une analyse du vocabulaire de base, quelques traits morphologiques et syntaxiques. En outre, un article plus complet a été publié en russe (Aplonova \& Vydrin 2017).

3 Dans cet article, je suis l'orthographe utilisée dans l'article précité. Notamment, les tons sont marqués par des accents : le double accent aigu (ă) désigne le ton extra-haut ; un accent aigu (á), le ton haut ; l'accent grave (â), le ton bas; le double accent grave (â), 
le ton extra-bas. Le point au-dessous des voyelles $e$ et $o(e, o)$ désigne l'articulation avec la racine de langue retraitée (-ATR), un trait qui est phonologique en goo. La nasalization est marquée par la tilde au-dessous de la voyelle.

4 Cette étude est basée sur les données recueillies au cours de deux missions de terrain en Côte d'Ivoire, en août-septembre 2015 et en janvier 2017. J'ai travaillé avec un seul assistant, Goo Roger Douané, originaire du village Zagwę. J'ai utilisé les questionnaires typologiques d'Östen Dahl (Dahl 1985; Dahl 2000) et d'Andrey Shluinsky adaptés aux réalite-'s africaines; un petit corpus de textes oraux spontanés a été exploité également. Ces textes ont été enregistrés, transcrits, traduits et annotés dans le programme Toolbox. Les auteurs de ces textes sont aussi originaires de Zagwę. Les exemples issus de ces textes sont dotés des références; la liste des textes est donnée à la fin de l'article.

5 L'article comporte deux parties. Dans la première, une description des éléments morphologiques exprimant les valeurs grammaticales verbales sera présentée, notamment les éléments pronominaux (2.1), les modifications tonales (2.2), les suffixes (2.3) et les auxiliaires (2.4). Dans la deuxième, les constructions verbales seront analysées avec un accent sur la concurrence des constructions de l'aoriste et du parfait dans les textes naturels.

\section{Éléments morphologiques exprimant les valeurs grammaticales verbales}

\section{Éléments pronominaux}

6 Dans les langues mandé-sud, les valeurs du TAM et de la polarité sont exprimées non seulement dans la forme verbale, mais aussi (et surtout) par des éléments pronominaux. Par exemple, afin de former une construction du parfait (1a), il faut utiliser un élément pronominal particulier, tandis que la forme du verbe reste la même que celle de la construction de l'imparfait négatif (1b) ou de l'impe-'ratif (1c).

\begin{tabular}{|l|l|l|}
\hline$(1 \mathrm{a})$ & $\mathcal{B a}_{\sim}^{\prime \prime}$ & bli. \\
\hline & 1SG.PRF & sauter \\
\hline
\end{tabular}

7 'J'ai sauté'.

\begin{tabular}{|l|l|l|}
\hline (1b) & Băa a & bli. \\
\hline & 1SG.IPFV.NEG & sauter \\
\hline
\end{tabular}

8 'Je ne saute pas'.

9 (1c) Blì! 'Saute!'

10 Les éléments pronominaux ont un paradigme flexionnel riche. Ils se subdivisent en deux groupes: pronoms sujets (Tableau 1) et marques pronominales prédicatives (MPP, 
Tableau 2). Les pronoms sujets sont en distribution complémentaire avec les groupes nominaux (GN) complets $(2 \mathrm{a}, \mathrm{b})$, tandis que les MPP ne le sont pas; elles doivent apparaître même en présence d'un GN sujet $(3 a, b)$.

\begin{tabular}{|l|l|l|l|l|}
\hline$(2 \mathrm{a})$ & $\grave{E}$ & $y_{\text {ă }}^{\prime \prime}$ & páá & bä. \\
\hline & 3SG.EXI & œil & lancer:3SG & sur \\
\hline
\end{tabular}

11 'Il le voit'.

\begin{tabular}{|l|l|l|l|l|l|}
\hline$(2 \mathrm{~b})$ & Búgá & $k \ddot{\gamma}$ & $y^{\prime \prime}$ & páá & bä. \\
\hline & Bouga & MP & $\propto i l$ & lancer:3SG & sur \\
\hline
\end{tabular}

12 'Bouga le voit'.

13 Dans (2a), un pronom sujet ẹ de la série existentielle est utilisé, tandis que dans (2b) le sujet est représenté par un groupe nominal complet, et le pronom sujet est absent.

\begin{tabular}{|l|l|l|l|l|}
\hline$(3 \mathrm{~A})$ & Yà & $y_{a}^{\prime \prime}$ & páá & bä. \\
\hline & 3SG.PRF & œil & lancer:3SG & sur \\
\hline
\end{tabular}

14 'Il l'a vu'.

\begin{tabular}{|l|l|l|l|l|l|}
\hline$(3 b)$ & Búgá & yà & $y_{\text {aá }}^{\prime \prime}$ & páá & bä. \\
\hline & Bouga & 3SG.PRF & œil & lancer:3SG & sur \\
\hline
\end{tabular}

15 'Bouga l'a vu'.

16 Dans (3a), une MPP yà de la série du parfait est utilisée ; elle est obligatoire même en présence d'un groupe nominal sujet (3b).

Tableau 1. Pronoms sujets

\begin{tabular}{|c|c|c|c|c|c|c|c|}
\hline \multirow{2}{*}{ Série } & \multicolumn{3}{|c|}{ SG } & \multicolumn{4}{|l|}{ PL } \\
\hline & 1 & & 3 & 1 & & 2 & 3 \\
\hline Existentiel, EXI & ä & & $\grave{e}, y \grave{a}$ & kọ & & $k a$ & wọ, wa \\
\hline Immediat, IMM & $a ́$ & & $\ddot{e}$ & kọ & & $k a ́$ & wo \\
\hline Impératif, IMP & - & $\varnothing$ & - & kộ (incl.) & kọoộ (excl.) & $k \ddot{a}$ & - \\
\hline
\end{tabular}




\begin{tabular}{|c|c|c|c|c|c|c|}
\hline Subjonctif, sUBJ & a & |"i & $\ddot{e}$ & kọ & $k a$ & wọ \\
\hline Conditionnel, COND & $a ́$ & $\hat{i}$ & $y \ddot{a}$ & kó & $k a ́$ & wä \\
\hline
\end{tabular}

Tableau 2. Marques pronominales prédicatives (MPP)

\begin{tabular}{|c|c|c|c|c|c|c|}
\hline \multirow[t]{2}{*}{ Série } & \multicolumn{3}{|l|}{ SG } & \multicolumn{3}{|l|}{ PL } \\
\hline & 1 & 2 & 3 & 1 & 2 & 3 \\
\hline Parfait, PRF & $6 \stackrel{a}{\prime \prime}$ & $b_{a}^{\prime \prime}$ & $y \grave{a}$ & $k w a a_{-: 1}^{\prime \prime}$ & $k a ̈$ & $w a i$ \\
\hline Prospectif, PROS & $6 \stackrel{\sim}{a} a \mathfrak{a}$ & biii & ẹe & kọọo & kàá & wo ó \\
\hline Imparfait négatif, IPFV.NEG & $6 \stackrel{\sim}{\prime \prime \prime \prime \prime}$ & báa & yäá & kwa & káa & wäá \\
\hline Négatif, NEG & $n$ & $\hat{i}$ & $\ddot{e}$ & kọ & $k a ́$ & wọ̆ \\
\hline
\end{tabular}

17 Le pronom existentiel de la 3ème personne singulier ẹ fusionne régulièrement avec le pronom non-sujet de la 3ème personne singulier ä en fonction de complément d'objet direct, ce qui donne une forme contractée yà (4).

\begin{tabular}{|c|c|c|c|c|c|c|}
\hline (4) & Yà & sí-á & $6 \ddot{~}$ & $\grave{e}$ & lú-âa & kä. \\
\hline & 3SG.EXI:3SG & prendre-INF1 & puis & 3SG.EXI & venir $\backslash \mathrm{T} 1-\mathrm{INF} 1: 3 \mathrm{SG}$ & avec \\
\hline
\end{tabular}

18 'Elle l'a pris, puis elle l'a apporte_'́.' [150812_Gbo_Maslo_Yo_Prizrak(Part1).018]

19 En (4), le prédicat de la première proposition est un verbe transitif sí 'prendre' qui exige un objet direct formel, même si du point de vue sémantique on n'en a pas besoin. Pourtant, vu que le sujet est exprimé par le pronom existentiel de 3ème personne du singulier ẹ, une forme contractée est utilisée.

Dans la série imperative les pronoms de la 1ère personne du pluriel s'opposent par la clusivité : un pronom inclusif est présenté en (5), et un pronom exclusif en (6).

\begin{tabular}{|l|l|l|l|}
\hline$(5)$ & Kộ & lō & gbü̈̋gíl \\
\hline & 1PL.IMP.INCL & aller & à.la.maison \\
\hline
\end{tabular}

21 'Allons à la maison!' (une invitation à l'interlocuteur à partir ensemble).

\begin{tabular}{|l|l|l|l|}
\hline (6) & Kọọo & ló & gbưuǵgí! \\
\hline & 1PL.IMP.EXCL & aller & à.la.maison \\
\hline
\end{tabular}



(présentés dans les colonnes «Contour tonal 1 » et «Contour tonal $2 »$ du Tableau 3). Ainsi, les verbes se subdivisent en classes tonales (Tableau 3) en fonction de leur structure segmentale et leur contour tonal lexical.

Tableau 3. Les classes tonales des verbes

\begin{tabular}{|l|l|l|l|l|l|}
\hline Classe & $\begin{array}{l}\text { Structure } \\
\text { segmentale }\end{array}$ & $\begin{array}{l}\text { Contour tonal } \\
\text { lexical }\end{array}$ & $\begin{array}{l}\text { Contour tonal } \\
1\end{array}$ & $\begin{array}{l}\text { Contour tonal } \\
2\end{array}$ & $\begin{array}{l}\text { Nombre des } \\
\text { verbes }\end{array}$ \\
\hline 1 & CV & eB & H & H & 36 \\
\hline 2 & CVV & eB-eB & B-B & B-(B) & 49 \\
\hline 3 & CVV & H-H & B-B & H-(H) & 9 \\
\hline 4 & CVV & eH-H & B-B & eH-eH & 21 \\
\hline 5 & CV & H & H & eH & 33 \\
\hline 6 & CVlV & B-H & B-H & B-eH & 4 \\
\hline
\end{tabular}

Note. Abréviations: $\mathrm{C}$, consonne ; $\mathrm{V}$, voyelle ; $\mathrm{eB}$, ton extra-bas ; $\mathrm{B}$, ton bas ; $\mathrm{H}$, ton haut ; $\mathrm{eH}$, ton extra-haut. Dans la dernière colonne, le nombre de verbes par classe est indiqué (en se basant sur un dictionnaire goo dont la version actuelle comporte 1013 lexèmes, y compris 152 verbes).

Dans la plupart des constructions verbales, le verbe apparaît avec son contour tonal lexical sans suffixes. Parmi les constructions affirmatives, il s'agit de celles du parfait 
(20), du prospectif (24), de l'imperifratif (31), du subjonctif (28) ; parmi les constructions négatives, ce sont celles de l'imperfectif (42), du perfectif (44) et du subjonctif (45). Le contour tonal lexical peut être remplacé par le contour tonal 2, sauf si le verbe apparaît dans la position finale dans la phrase.

Le verbe assume le contour tonal 1 à l'habituel (14) et dans l'aoriste, dans ce dernier cas il s'adjoint en même temps le suffixe d'infinif-1 (18). Le contour tonal 2 se combine toujours avec des suffixes et des clitiques. Il apparait dans les constructions affirmatives du progressif (15), du résultatif (33), du passé et du futur immédiat $(34,35)$, du futur (23) et dans les constructions négatives correspondantes. De plus, le verbe dans la construction du prohibitif (46) a un contour tonal 2 également.

\section{Suffixes}

Le goo possède des formes suffixées suivantes: trois formes d'infinitif, le supin et le gérondif.

L'infinitif, le gérondif et le supin sont d'abord des formes verbales non-finies. Cependant, en goo, ils peuvent apparaître également en fonction des verbes finis (cf. Tableau 4). On dirait que pour le goo, une interprétation du caractère fini comme caractéristique graduelle de la proposition entière (Givón 2001:25-26) conviendrait mieux qu'une opposition binaire des formes verbales.

Tableau 4. Formes suffixées

\begin{tabular}{|c|c|c|c|}
\hline Forme & Structure & Constructions finies & Emplois non-finis \\
\hline ge-'rondif & $\mathrm{V} \backslash \mathrm{T} 2-\grave{e}$ & résultative & $\begin{array}{l}\text { action accompagnante (8), nominalisation phrastique } \\
\text { (9) }\end{array}$ \\
\hline infinitif-1 & $\mathrm{V} \backslash \mathrm{T} 1-a ́$ & de l'aoriste & actant sentenciel du verbe bọ 'terminer' (10) \\
\hline infinitif-2 & $\mathrm{V} \backslash \mathrm{T} 2-\ddot{a}$ & $\begin{array}{ll}\mathrm{du} & \text { futur, } \\
\text { prohibitive } & \end{array}$ & actant sentenciel du verbe ź̃ 'commencer' (11) \\
\hline infinitif-3 & $\mathrm{V} \backslash \mathrm{T} 2-l a ̈$ & progressive & actant sentenciel du verbe to ' 'laisser/rester' (40) \\
\hline supin & $\mathrm{V} \backslash \mathrm{T} 2-\ddot{i}$ & immédiate & prédication seconde à valeur de but (12) \\
\hline
\end{tabular}

Abréviations : T1, contour tonal 1; T2, contour tonal 2 (cf. Tableau 3).

\begin{tabular}{|l|l|l|l|l|l|}
\hline$(8)$ & $K^{\prime \prime}$ & ló-lä & ń & gàa-ẹ & kä. \\
\hline & 2PL.EXI & aller\T2-INF3 & 1SG & regarder-GER & avec \\
\hline
\end{tabular}

'Vous êtes en train de me regarder en marchant'.

\begin{tabular}{|c|c|c|c|c|c|}
\hline 9) Lïä́n & $z \ddot{n}$ & $\hat{i}$ & $1 n ̃-\ddot{e}$ & $k{ }^{\prime \prime}$ & gbẹẹe \\
\hline
\end{tabular}




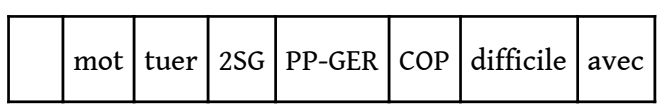

'Il est difficile de parler avec toi'.

\begin{tabular}{|l|l|l|l|}
\hline$(10)$ & Yà & bọ & wŕsi i-áa \\
\hline & 3SG.PRF & arrêter & pleurer-INF1 \\
\hline
\end{tabular}

'Il a arrêté de pleurer'.

\begin{tabular}{|l|l|l|l|}
\hline$(11)$ & $\grave{E}$ & $z \hat{z}-\tilde{a}$ & wช́sí-ä. \\
\hline & 3SG.EXI & commencer-INF1 & pleurer\T2-INF2 \\
\hline
\end{tabular}

‘Il a commencé de pleurer'.

\begin{tabular}{|l|l|l|l|l|l|}
\hline$(12)$ & $\grave{E}$ & ló-á & $y \ddot{i}$ & gùlù & sí-ï. \\
\hline & 3SG.EXI & aller-INF1 & eau & trou & prendre\T2-SUP \\
\hline
\end{tabular}

'Il est allé puiser de l'eau'.

\section{Auxiliaires}

Les auxiliaires sont des marques prédicatives qui expriment des valeurs de TAM et de polarité mais n'ont pas de valeurs pronominales, telles que la personne et le nombre.

Les origines de certaines marques prédicatives sont assez transparentes: ce sont des verbes grammaticalisés. Ainsi, gọ̆ (qui provient du verbe gọ́ 'partir') et lö (qui provient du verbe ló 'aller') forment les constructions immédiates, gúu (gưuá ) 'être dans le passé' forme une construction d'ultérieur et celle du présomptif (41), tựà 'continuer' forme une construction du continuatif (39). Le verbe auxiliaire gọ à ton haut apparaît dans la construction du prohibitif.

Le verbe gú 'être dans le passé' change sa forme en fonction de la construction. Dans la construction de l'ultérieur, il a deux variantes équivalentes : gú (le contour tonal de base) et gúáa (le contour tonal de base + suffixe de l'infinitif 1 -á). Dans le présomptif, il assume un contour tonal extra-bas. Actuellement, ce verbe fonctionne uniquement comme un auxiliaire. Pourtant, dans le dan-gouèta, son homologue gũ s'emploie comme un verbe lexical également.

Quant à tữa 'continuer', il est fort probable que cette forme comporte un suffixe (tü + ä), ce qui est conforté par l'existence en dan-gouèta d'un verbe apparenté tü 'continuer, être encore'.

41 La marque négative kä, qui apparaît dans de nombreuses constructions négatives (cf. 2.2.3), remonte à la marque négative ká du Proto-Dan-Gouro. L'origine de la marque 
affirmative imperfective $k \not ̛$ utilisée auprès du sujet exprimé par un groupe nominal complet (13) a été l'objet d'une étude détaillée par Thomas Bearth (1995).

\begin{tabular}{|c|c|c|c|c|c|}
\hline (13a) & Gběćn-bọ & $k \ddot{\gamma}$ & wì & wع̋"z-bọ & 6̆̀̀̆. \\
\hline & chien-PL & MP & viande & os-PL & manger $\backslash \mathrm{T} 1$ \\
\hline
\end{tabular}

'Les chiens mangent des os'.

\begin{tabular}{|l|l|l|l|l|}
\hline (13b) & Wo - & wìi & wě̋́-bọ & bhr̀r̀. \\
\hline & 3PL.EXI & viande & os-PL & manger \T1 \\
\hline
\end{tabular}

'Ils mangent des os'.

En plus, il y a deux lexèmes, lú 'déjà' et kưú 'ne ... plus', qui apparaissent obligatoirement dans les constructions du présomptif (lús) et du continuatif (kưuúu). Apparemment, ils sont d'origine adverbes. Pourtant, le fait qu'ils doivent être utilisés obligatoirement dans ces constructions empêche de les attribuer à la classe des adverbes, donc, la question de leur appartenance à une partie de discours reste ouverte.

\section{Constructions verbales}

Tableau 5. Constructions verbales du goo

\begin{tabular}{|c|c|c|}
\hline Construction verbale & Affirmative & Négative \\
\hline Habituel & $\mathrm{EXI}-(0)-\mathrm{V} \backslash \mathrm{T} 1$ & IPFV.NEG $-(0)-V$ \\
\hline Progressif & $\mathrm{EXI}-(0)-\mathrm{V} \backslash \mathrm{T} 2-l_{a}$ & IPFV.NEG - (0) - V \T2-lä \\
\hline Passé immédiat & IMM - gộ- (O) - V\T2-SUP & NEG - kä - gộ- (O) - V\T1-SUP \\
\hline Aoriste & $\mathrm{EXI}-(\mathrm{O})-\mathrm{V} \backslash \mathrm{T} 1-\mathrm{INF} 1$ & \multirow{2}{*}{$\mathrm{NEG}-k^{\prime \prime}-(0)-\mathrm{V}$} \\
\hline Parfait & $\mathrm{PRF}-(0)-\mathrm{V}$ & \\
\hline Résultatif & $\mathrm{EXI}-\mathrm{V} \backslash \mathrm{T} 2-\mathrm{GER}-k_{a}^{\prime \prime}$ & IPFV.NEG $-(\mathrm{O})-\mathrm{V} \backslash \mathrm{T} 2$-GER $k a ̈$ \\
\hline Futur & $\mathrm{EXI}-(\mathrm{O})-\mathrm{V} \backslash \mathrm{T} 2$-INF2 & \multirow{2}{*}{ IPFV.NEG $-(0)-V \backslash T 2$-INF2 } \\
\hline Prospectif & PROS $-(0)-V$ & \\
\hline Futur immédiat & IMM - lö - (0) - V\T2-SUP & $\mathrm{NEG}-k^{\prime \prime}-l o-1-(0)-\mathrm{V} \backslash \mathrm{T} 1$-SUP \\
\hline Subjonctif & SUBJ $-(0)-V$ & $N E G-g o ́ n-(0)-V$ \\
\hline
\end{tabular}




\begin{tabular}{|c|c|c|}
\hline Impératif/Prohibitif & IMP $-(0)-V$ & $\mathrm{NEG}-g o ̣-(0)-\mathrm{V} \backslash \mathrm{T} 2$-INF2 \\
\hline Continuatif & EXI tu $a-(0)-V \backslash T 2-I N F 2$ & IPFV.NEG tu \\
\hline Présomptif & $\mathrm{PRF}-g \ddot{\tilde{u}}-(0)-\mathrm{V} l \tilde{u}$ & \\
\hline Ultérieur & EXI gú $/ g \tilde{u} \tilde{a}-(0)-V \backslash \mathrm{T} 2-\mathrm{INF} 2$ & \\
\hline
\end{tabular}

Formellement, on peut distinguer les constructions de base et les constructions périphrastiques, mais elles sont au même plan en ce qui concerne leur sémantisme grammatical. Les constructions négatives sont moins nombreuses que celles affirmatives. Elles ne sont pas toujours en parallèle avec les constructions affirmatives, et pour cette raison elles seront examinées séparément.

\section{Constructions affirmatives de base}

46 L'habituel décrit l'action qui se produit d'habitude et qui tend à devenir une caractéristique du sujet. La série existentielle des pronoms sujets est employée, et là où le sujet n'est pas pronominalisé, la marque prédicative $k \ddot{\gamma}$ apparaît. Le verbe assume le contour tonal 1.

\begin{tabular}{|l|l|l|l|}
\hline$(14)$ & E@ & biti & gí-wó. \\
\hline & 3SG.EXI & riz & l'intérieur-faire $\backslash T 1$ \\
\hline
\end{tabular}

47 [Qu'est-ce que ton frère fait le matin d'habitude?] 'Il désherbe le riz'.

48 Le progressif renvoie à une situation concomitante soit avec le moment de la parole, soit avec un autre événement qui sert de point de repère temporel. La série existentielle des pronoms sujets est employée ; si le sujet est exprimé, il est suivi de la marque prédicative $k \not \gamma$. Le verbe assume le contour tonal 2 et s'adjoint le suffixe de l'infinitif 3, -lä.

\begin{tabular}{|c|c|c|c|c|c|c|}
\hline (15) & Tìa & $k \ddot{\gamma}$ & wéé & bi-lă & maki & gí. \\
\hline & Tia & MP & vin & boire \T2-INF3 & maquis & dans \\
\hline
\end{tabular}

'[Maintenant] Tia est en train de boire du vin au maquis'.

Le progressif peut être employé dans la construction de l'ultérieur.

\begin{tabular}{|c|c|c|c|c|c|}
\hline$(16)$ & $\dot{E}$ & $g u-a$ & gíyä & $z 0$ & $y \varepsilon ̋ \varepsilon$ \\
\hline & 3SG.EXI & être.dans.le.passé-INF1 & manioc & pile \T2-INF2 & hier \\
\hline
\end{tabular}




\begin{tabular}{|l|l|l|l|l|l|}
\hline$k \gamma^{\prime}$ & Tìa & $k$ r̆ & lẹe & $z$ ź-lä & bệi. \\
\hline CNJ & Tia & MP & endroit & tuer \T2-INF3 & au.champ \\
\hline
\end{tabular}

\section{'Dépêche-toi ! Le bus va partir !'}

L'aoriste renvoie aux actions accomplies. La construction de l'aoriste comporte soit un pronom de la série existentielle, soit, si le sujet est exprimé, la marque prédicative kð̋ dans la position post-sujet. Le verbe assume le contour tonal 1 et s'adjoint le suffixe de l'infinitif-1 -á. Ainsi, l'exemple (18) peut avoir deux significations différentes : soit le chef reste toujours là où il est arrivé, soit il est déjà parti.

\begin{tabular}{|l|l|l|l|}
\hline (18) & Lüütíi & $k \not ̋$ & lú-ạ. \\
\hline & chef.de.village & MP & venir \T1-INF1 \\
\hline
\end{tabular}

\section{'Le chef du village est arrivé'. 1) On peut le voir maintenant. 2) Il est déjà reparti.}

On peut mentionner son incompatibilité avec l'adverbe lú ‘déjà'.

\begin{tabular}{|c|c|c|c|c|c|c|}
\hline (19) & *Ń & | & $k \ddot{\gamma}$ & gwiä & $z \mathcal{N}_{\alpha}^{\prime a ́}$ & lú. \\
\hline & $1 \mathrm{SG}$ & jeune.sibling & MP & manioc & piler $\backslash \mathrm{T} 1-\mathrm{INF} 1$ & déjà \\
\hline
\end{tabular}

7 (le sens attendu: 'Ma soeur cadette a déjà pilé le manioc'.)

Le parfait renvoie à une action dont l'effet a une incidence sur la situation actuelle. Il se forme par les MPP de la série du parfait; le verbe maintient son contour tonal lexical. Contrairement à (18), l'exemple (20) ne peut se référer qu'à une situation dont le résultat est toujours visible.

\begin{tabular}{|l|l|l|l|}
\hline$(20)$ & Lüütíi & $\grave{a}$ & lü. \\
\hline & chef.de.village & 3SG.PRF & venir \\
\hline
\end{tabular}

'Le chef de village est arrivé' (et on peut le voir au moment de la parole).

La construction du parfait rend aussi le sens expérientiel qui désigne une action accomplie par le sujet au moins une fois dans sa vie. 


\begin{tabular}{|l|l|l|l|l|l|l|}
\hline$(21)$ & Bå & $y_{\sim}^{\prime \prime}$ & $p a ̈$ & $\ddot{a}$ & $6 a ̈$. & \\
\hline & 1 1SG.PRF & œil & lancer & $3 S G$ & sur & \\
\hline
\end{tabular}

61

\begin{tabular}{|c|c|c|c|c|c|c|}
\hline$*(22)$ & $B_{\tilde{\sim}}^{\prime \prime}$ & ya & $p \ddot{a}$ & $\ddot{a}$ & bä & zîä. \\
\hline & 1SG.PRF & œil & lancer & $3 S G$ & sur & souvent \\
\hline
\end{tabular}

63 (le sens attendu: 'J'ai été là-bas souvent'.)

64 La construction du futur exprime toutes les valeurs de la zone sémantique du futur. $\mathrm{Si}$ nécessaire, les adverbes temporaux peuvent être employés. La construction du futur comporte soit un pronom de la série existentielle, soit, si le sujet n'est pas pronominalisé, la marque prédicative $k \ddot{\gamma}$ dans la position post-sujet. Le verbe assume le contour tonal 2 et s'adjoint le suffixe de l'infinitif-2 -

\begin{tabular}{|c|c|c|c|c|}
\hline (23) & $A$ & gẹẹ & $k 0$ ìn & $z 0$ \\
\hline & 1SG.EXI & banane & boule & piler \T2-INF2 \\
\hline
\end{tabular}

65 '[Quand je reviens,] je pilerai le foutou³-banane'.

66 Le prospectif se réfère à une action pour le déroulement de laquelle toutes les conditions sont réunies. La construction prospective comporte une MPP de la série prospective ; le verbe porte son contour tonal leixical.

\begin{tabular}{|c|c|c|c|c|c|c|}
\hline (24) & |í & gọ̆ & gúyä & 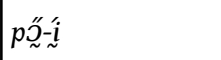 & biii & lưáa \\
\hline & 2SG.IMM & IMM.PST & manioc & creuser $\backslash \mathrm{T} 2$-SUP & 2SG.PROS & venir:3SG \\
\hline
\end{tabular}

\begin{tabular}{|l|l|l|}
\hline kă & $b^{\prime \prime i}$ & küán. \\
\hline avec & 2SG.PROS & éplucher \\
\hline
\end{tabular}

67 'Dès que tu as dé-'́terré une racine de manioc, tu l'apportes et tu l'épluches'. [150808_RG_foutou.002]

68 Le prospectif est difficilement compatible avec les circonstants se référant au futur plus ou moins éloigné.

\begin{tabular}{|l|l|l|l|l|l|l|}
\hline (25) & ?"Bàá & ló & bệi & loóo & ziàà & pièé. \\
\hline
\end{tabular}




\begin{tabular}{|l|l|l|l|l|l|l|l|}
\hline & 1SG.PROS & aller & au.champ & semaine & plus.tard:3SG & PP \\
\hline
\end{tabular}

\begin{tabular}{|c|c|c|c|c|c|c|c|}
\hline (26) & Ń & doọọ & ẹẹ & $6 \grave{a}$ & kwiii & looódó & tö. \\
\hline & $1 S G$ & aînéné & 3SG.PROS & POSS & maison & vendre & maintenat \\
\hline
\end{tabular}

\begin{tabular}{|c|c|c|c|c|c|c|c|c|c|}
\hline (27) & Ẹé & kwíi & $y \varepsilon ́$ & $s i$ & $k \dot{\gamma}^{\prime}$ & $y \ddot{a}$ & dàa & $\ddot{a}$ & bä. \\
\hline & 3SG.PROS & peau & ce & prendre & $\mathrm{CNJ}$ & 3SG.SUBJ:3SG & entrer & $3 S G$ & PP \\
\hline
\end{tabular}

72 'Elle veut prendre cette peau pour la mettre sur elle'. [150812_Gbo_DeuxFemmes.046]

73 Le subjonctif est utilisé dans une proposition autonome pour exprimer des valeurs modales différentes : l'ordre (28), la suggestion (29).

\begin{tabular}{|c|c|c|c|c|c|c|}
\hline (28) & $K \dot{\gamma}$ & $" i$ & bọ & tåă & $6 i-a$ & 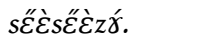 \\
\hline & $\mathrm{CNJ}$ & 2SG.SUBJ & finir & tabac & boire $\backslash \mathrm{T} 2$-INF2 & maintenant.INT \\
\hline
\end{tabular}

74 'Arrête de fumer tout de suite !'

\begin{tabular}{|l|l|l|l|}
\hline$(29)$ & Kọ & dì̀ & ẹ ? \\
\hline & 1PL.SUBJ & balader & Q \\
\hline
\end{tabular}

'Promenons-nous!'

Il est utilisé également dans les propositions subordonnées à valeur de but introduites par la conjonction $k \dot{\gamma}$.

\begin{tabular}{|c|c|c|c|c|c|c|c|}
\hline (30) & Ǎáa & pié & $k \gamma^{\prime}$ & $" i$ & gêe & kذָِ̀ & $z$ zె. \\
\hline & 1SG.EXI:3SG & désir & $\mathrm{CNJ}$ & 2SG.SUBJ & banane & boule & piler \\
\hline
\end{tabular}

77 'Je veux que tu prépares le foutou-banane'.

78 La construction subjonctive comporte un pronom sujet de la série subjonctive; le verbe apparaît avec son contour tonal lexical. 
L'impe-'ratif exprime les valeurs d'injonction. À cause du sémantisme de l'impératif, le sujet d'une construction impérative ne peut être que pronominal, et au singulier le sujet ne peut pas être exprimé ouvertement (31). Par contre, le sujet pluriel doit être exprimé (32). Le verbe apparaît avec son contour tonal lexical.

\begin{tabular}{|l|l|l|l|}
\hline$(31)$ & $\varnothing$ & lèẹ & sálaáa! \\
\hline & 2SG.IMP & endroit & nettoyer \\
\hline
\end{tabular}

'Nettoie ici !'

\begin{tabular}{|l|l|l|l|}
\hline (32) & Kä & lèẹ & săläă ! \\
\hline & 2PL.IMP & endroit & nettoyer \\
\hline
\end{tabular}

'Nettoyez ici!'

\section{Constructions affirmatives périphrastiques}

Le résultatif désigne une situation qui présuppose une action précédente (Nedjalkov 1988:7). La construction résultative est formée par un pronom existentiel ou, si le sujet n'est pas pronominalisé, par la copule $k \ddot{\gamma}$; le verbe assume le contour tonal ascendant et adjoint le suffixe de gérondif -ẹ ; La forme verbale est suivie de la postposition kä.

\begin{tabular}{|c|c|c|c|c|c|c|}
\hline (33) & Báà & läkëlé & wžह & $k \ddot{~}$ & yह̋"z-ẹ & kä. \\
\hline & 1SG.POSS & cle & os & COP & casser\T2-GER & avec \\
\hline
\end{tabular}

'Ma cles's est cassée'.

La construction du passé immédiat exprime une action qui vient de se produire. Cette construction est formée par un pronom existentiel ou, si le sujet n'est pas pronominalisé, par la marque prédicative $k \widehat{\gamma}$; le pronom sujet ou la copule est suivi(e) par le verbe auxiliaire gọ ; le verbe significatif assume le contour tonal ascendant et adjoint le suffixe de supin $-{ }^{\prime \prime}$.

\begin{tabular}{|c|c|c|c|c|}
\hline (34) & $\ddot{E}$ & gọ & pỡ & $6 \grave{\gamma}-\hat{i}$. \\
\hline & 3SG.IMM & IMM.PST & chose & manger $\backslash \mathrm{T} 2$-SUP \\
\hline
\end{tabular}

'Il vient de manger'.

Le futur immédiat renvoie à une action qui va se dérouler dans un avenir proche par rapport au moment de la parole. Cette construction est formée par un pronom existentiel ou, si le sujet n'est pas pronominalisé, par la marque prédicative $k \not{\gamma}$; le 
pronom sujet ou la copule est suivi(e) par le verbe auxiliaire lö; le verbe significatif assume le contour tonal 2 et adjoint le suffixe de supin -î.

\begin{tabular}{|l|l|l|l|l|l|}
\hline$(35)$ & A & lo & Gälä & $k \varepsilon^{\prime \prime}-i$ & bệi. \\
\hline & 1SG.IMM & IMM.FUT & travail & faire-SUP & au.champ \\
\hline
\end{tabular}

87 'Je vais travailler au champ'.

88 Cette construction désigne l'événement proche du moment de la parole. Cf. cependant l'exemple (36) où l'événement n'est pas immédiat en termes de la distance temporelle absolue $^{4}$, cependant c'est la construction du futur immédiat qui est utilisée. Il s'avère que «la semaine prochaine» est incluse par le locuteur dans le domaine du futur proche.

\begin{tabular}{|l|l|l|l|l|}
\hline (36) & $\AA$ & lö & loôi & bệi \\
\hline & 1SG.IMM & IMM.FUT & aller\T2-SUP & au.champ \\
\hline
\end{tabular}

\begin{tabular}{|l|l|l|}
\hline loó & zìàa & piée. \\
\hline semaine & plus.tard:3SG & PP \\
\hline
\end{tabular}

'Je vais aller au champ la semaine prochaine'.

90 La construction du futur immédiat peut exprimer également les valeurs modales d'assertion (37) et d'obligation (38).

91 (37) Á lò lơi bệi. ‘[C'est sûr que] je vais aller au champ’.

\begin{tabular}{|c|c|c|c|c|}
\hline (38) & Í & lö & $k \grave{j}$ & zu lülí. \\
\hline & 2SG.IMM & IMM.FUT & bras & laver\T2-SUP \\
\hline
\end{tabular}

'Tu dois laver tes mains'.

La construction continuative exprime la continuation de l'action ou l'état. Cette construction est formée par un pronom existentiel ou, si le sujet n'est pas pronominalisé, par la marque prédicative $k \ddot{\gamma}$; le pronom sujet ou la copule est suivi(e) par le verbe auxiliaire tưa ; le verbe significatif assume le contour tonal ascendant et s'adjoint le suffixe de l'infinitif-2.

\begin{tabular}{|c|c|c|c|c|c|c|c|c|}
\hline (39) & $A$ & yấ & yääliẹ & páá & $6 \ddot{a}$ & $k \dot{\gamma}^{\prime}$ & $\grave{e}$ & tua \\
\hline & $1 \mathrm{SG}$ & œil & fois & lancer-INF1:3SG & sur & $\mathrm{CNJ}$ & 3SG.EXI & CONT \\
\hline
\end{tabular}




\begin{tabular}{|l|l|l|l|}
\hline$\dddot{a}$ & $6 \grave{a}$ & $k w$ wii & dó-ä. \\
\hline 3SG & POSS & maison & construire-INF2 \\
\hline
\end{tabular}

\section{Constructions négatives}

En fonction du contexte, l'imperfectif négatif peut exprimer les valeurs négatives de l'habituel (43) et du futur (42). Il se forme à l'aide des MPP imperfectives négatives; le verbe apparait dans sa forme lexicale.

\begin{tabular}{|c|c|c|c|c|}
\hline (42) & Kwää & Gälá & $k \varepsilon \varepsilon^{\prime}$ & tìli. \\
\hline & 1PL.IPFV.NEG & travail & faire & demain \\
\hline
\end{tabular}

'Nous ne travaillons pas demain'.

\begin{tabular}{|l|l|l|l|}
\hline$(43)$ & Băá & wò̀̀ & $6 \underset{\sim}{a}$. \\
\hline & 1SG.IPFV.NEG:3SG & voix & entendre \\
\hline
\end{tabular}

101 [Il parle toujours très bas] 'Je ne l'entends pas'.

102 L'homologue négatif du parfait et de l'aoriste est le perfectif négatif. Il se forme à l'aide des MPP perfectives négatives et l'opérateur kä qui suit la MPP. 


\begin{tabular}{|l|l|l|l|l|}
\hline$(44)$ & $\ddot{E}$ & $k a ̈$ & $l \tilde{u}$ & dọ. \\
\hline & 3SG.NEG & NEG & venir \T2 & jamais \\
\hline
\end{tabular}
l'aoriste (capable à exprimer n'importe quelle action accomplie), et les donné d'élicitation ne suffisent pas pour l'analyse de la distribution des deux constructions. Dans ce chapitre seront traitées les données provenant de textes oraux transcrits et annotés. Actuellement, le volume de ce corpus est assez modeste, environ 400 propositions, ce qui correspond approximativement à 20 minutes de l'enregistrement 
audio. Néanmoins, même si l'échantillon est limité, il est possible de dégager certaines tendances de l'usage des deux constructions.

Dans cette analyse, je m'inspire beaucoup d'une étude similaire accomplie par Maria Khachaturyan (2016). Toutes les utilisations de l'aoriste et du parfait dans les textes analysés ont été marquées par deux paramètres : le type de sujet dans les constructions de l'aoriste/ parfait (GN / pronom ou MPP), et le type de la proposition (principale/ subordonnée) où apparaît la construction aoriste/ parfait. Le nombre total d'occurrences de ces constructions est 155, dont 108 sont des constructions du aoriste et 47 sont celles du parfait.

113 Le Tableau 6a présente la répartition des deux constructions selon le type de sujet.

Tableau 6a. Le type de sujet dans les constructions du aoriste et du parfait ${ }^{6}$

\begin{tabular}{|c|c|c|c|c|c|}
\hline & \multicolumn{2}{|c|}{ Aoriste } & \multicolumn{2}{|c|}{ Parfait } & \multirow{2}{*}{ TOTAL } \\
\hline & $\mathbf{N}$ & Fréquence & $\mathbf{N}$ & Fréquence & \\
\hline GN & 6 & 0,3 & 12 & 0,7 & 18 \\
\hline Pronom & 102 & 0,7 & 35 & 0,3 & 137 \\
\hline TOTAL & \multicolumn{2}{|l|}{108} & \multicolumn{2}{|l|}{47} & 155 \\
\hline
\end{tabular}

114 Le Tableau 6a (de même que le tableau 6b) est organisé comme suit: les colonnes fournissent des informations sur le nombre de constructions verbales de l'aoriste et du parfait, tandis que les lignes donnent le nombre des sujets exprimés par les GN et les pronoms. La colonne "Fréquence " montre le rapport du nombre de verbes dans les constructions dans lequelles le sujet est exprimé par un GN ou par un pronom, au nombre total de constructions verbales; constructions du parfait dans lequel le sujet est exprimé soit par un GN, soit par un pronom au nombre total de constructions verbales.

115 Selon les données présentées dans le Tableau 6a, la pronominalité du sujet est en corrélation avec le choix de la construction. Le sujet peut être exprimé par un GN avec une probabilité plus élevée dans la contruction du parfait que dans celle de l'aoriste. On peut supposer que cette distribution est due aux contextes typiques de l'apparition de l'aoriste, plutôt qu'à la forme du sujet. L'aoriste est la construction la plus utilisée dans le narratif où il est très typique d'avoir un protagoniste à qui on fait référence le plus souvent par le moyen des pronoms, plutôt que par les groupes nominaux complets.

Tous les textes disponibles sont des narrations. Cela veut dire que les actions mentionnées dans ces textes sont accomplies et ne sont plus d'actualité au moment de la parole, ce qui est typique de l'aoriste. Par contre, le parfait apparaît dans les textes pour décrire des événements en connexion avec le moment de parole. Cf. (49), où la situation est décrite pour la première fois: un soldat de la sorcière veut attaquer le guerrier par derrière et, à cet effet, il le prend de dos [le aoriste], alors Kiriku (le neveu du guerrier, le protagoniste de l'histoire) avertit son oncle que le guerrier l'a pris de dos [le parfait]. 


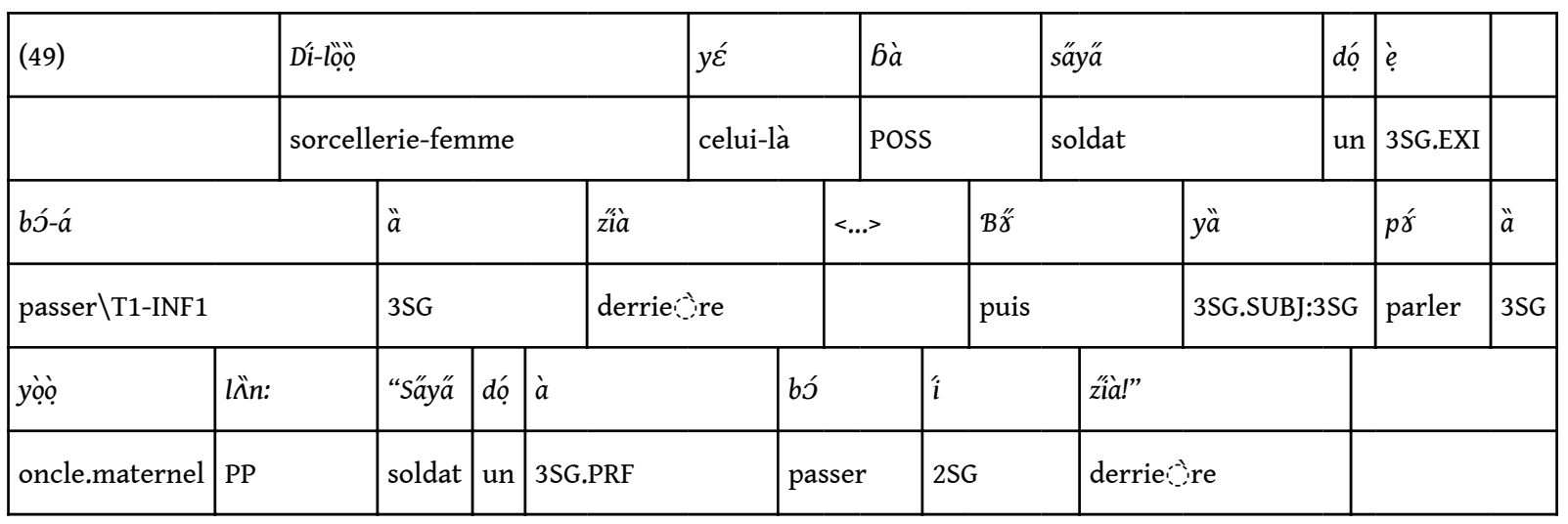

117 'L'un des soldats de la sorcière a pris le dos de l'(oncle maternel) [afin de le tuer], puis il (Kirkou) a dit à son oncle maternel : "Un soldat t'est passe 055-57].

118 Le Tableau $6 \mathrm{~b}$ présente la répartition des constructions du parfait et de l'aoriste selon le type de clause.

Tableau 6b. Types de propositions comportant les constructions du parfait et de l'aoriste ${ }^{7}$

\begin{tabular}{|l|l|l|l|l|l|}
\hline & \multicolumn{2}{|l|}{ Aoriste } & \multicolumn{2}{l|}{ Parfait } & \multirow{2}{*}{ TOTAL } \\
\cline { 2 - 5 } & N & Fréquence & N & Fréquence & \\
\hline Principale & 93 & 0,7 & 46 & 0,3 & 139 \\
\hline Subodonne-e & 15 & 0,9 & 1 & 0,1 & 16 \\
\hline TOTAL & \multicolumn{2}{|l|}{108} & 47 & $\mathbf{1 5 5}$ \\
\hline
\end{tabular}

119 Comme on peut le voir dans le Tableau 5b, la construction aoriste est utilisée dans les propositions subordonnées plus souvent que celle du parfait $(0,9$ contre 0,1$)$. Cf. (50), où le prédicat de la proposition subordonnée introduite par la conjonction lé est exprimé par la construction de l'aoriste.

\begin{tabular}{|c|c|c|c|c|c|c|c|}
\hline (50) & [LLñ-bọ & ló-á & $6 \grave{\tilde{a}} \grave{a}$ & gว̃ & lé] & wäá & kéć]. \\
\hline & enfant-PL & aller-INF1 & oiseau & PP & $\mathrm{CNJ}$ & 3PL.IPFV.NEG:3SG & faire \\
\hline
\end{tabular}

[Les enfants ont eu l'idée d'aller au champ pour chasser les oiseaux qui mangeaient les récoltes]. 'Quand les enfants sont arrivés, ils (les enfants) n'ont rien fait'. [150812_Gbo_DeuxFemmes.021]

121 Il est fort probable qu'une telle distribution reflète l'influence des facteurs non-formels qui puissent être plus importants et qui exigent une étude plus nuancée d'un corpus plus fourni. 
Textes spontanés

\begin{tabular}{|l|l|l|}
\hline Titre au Toolbox & Durée & Description \\
\hline 150812_Gbo_Maslo_Yo_Prizrak & 11:18 & $\begin{array}{l}\text { L'histoire d'un garçon qui était un batteur excellent et qui } \\
\text { est mort à cause du soleil. }\end{array}$ \\
\hline 150812_Gbo_DeuxFemmes & $4: 02$ & Un conte qui raconte une histoire de deux co-épouses. \\
\hline 150808_RG_foutou & $00: 50$ & Une recette de foutou. \\
\hline Kiriku & $07: 02$ & Un résumé du dessin animé « Kiriku ». \\
\hline
\end{tabular}

\section{Liste des abréviations}

$\backslash \mathrm{T} 1$ - modification tonale1

$\backslash \mathrm{T} 2$ - modification tonale 2

$\mathrm{CNJ}$ - conjonction

COP - copule

CONT - continuatif

EXI - série pronominale existentielle

GER - clitique de gérondif

GN - groupe nominal

IMM.PST - auxiliaire au passé immédiat

INCL - pronom inclusif

INF1 - suffixe de la première forme de l'infinitif

INF2 - suffixe de la deuxième forme de l'infinitif

INF3 - suffixe de la troisième forme de l'infinitif

IPFV.NEG - série imperfective négative des MPP

MP - marque prédicative

MPP - marque pronominale prédicative

$\mathrm{NEG}$ - série négative des MPP/opérateur négatif

ONM - onomatopée

POSS - série pronominale possessive/ postposition possessive

$\mathrm{PP}$ - postposition

PRF - série parfaite des MPP

PROH - auxiliaire du prohibitif

PROS - série prospective des MPP

PRSM - présomptif

SUBJ - série pronominale subjonctive

SUP - suffixe de supin 


\section{BIBLIOGRAPHY}

\section{Références}

Aplonova, Ekaterina \& Valentin Vydrin. 2017. Goo jazyk (гоо язык) [Goo]. In Valentin Vydrin, Yulia Mazurova, Anderj Kibrik \& Elena Markus (eds.), Jazyki mira: Jazyki mande (языки миРА: Языки МАНДЕ) [Languages of the world: Mande languages], 457-469. St. Petersburg: Nestor-Historia.

Bearth, Thomas. 1971. L'énoncé toura. Norman (Oklahoma): Société international de linguistique. Bearth, Thomas. 1995. Nominal periphrases and the origin of the predicative marker in Mande languages - an alternative view. Afrikanistische Arbeitspapiere 41. 89-117.

Dahl, Östen. 1985. Tense and aspect systems. Blackwell. Oxford.

Dahl, Östen. 2000. The grammar of future time reference in European Languages. In Georg Bossong \& Bernard Comrie (eds.), Tense and Aspect in the language of Europe, 309-328. Mouton de Gruyter. Berlin, New York.

Fedotov, Maksim. 2012. Konstrukcii immediatnogo predshestvovanija v iazyke gban. Nabroski k tipologii immediatnosti (КОНСТРУКЦИЯ ИММЕДИАТНОГО ПРЕДШЕСТВОВАНИЯ В ЯЗЫКЕ ГБАН. НАБРОСКИ К ТИПОЛОГИИ ИММЕДИАТНОСТИ) [Les constructions immédiates dans la langue gban. Une esquisse pour la typologie des constructions immédiates]. Problemy jazyka: Sbornik nauchnykh statej po materialam Pervoj knoferencii-shkoly "Problemy jazyka: vzgl'ad molodykh uchenykj” (Moskva, 20-22 sent'abr'a 2012) (ПРОБЛЕМЫ ЯЗЫКА: СБОРНИК НАУЧНЫХ СТАТЕЙ ПО МАТЕРИАЛАМ ПЕРВОЙ КОНФЕРЕНЦИИ-ШКОЛЫ «ПРОБЛЕМЫ ЯЗЫКА: ВЗГЛЯД МОЛОДЫХ УЧЕНЫХ» (МОСКВА, 20-22 СЕНТЯБРЯ 2012 Г., ИЯз РАн) [Problems of language: Collected papers following the 1st Conference-school "Problems of language: A vision by young scholars"], 316-343. Moscow: Institut jazykoznanija, Russian Academy of Sciences.

Givón, Talmy. 2001. Syntax: An Introduction. John Benjamins. Vol. II. Amsterdam, Philadelphia.

Khachaturyan, Maria. 2016. Semantics of perfective aspect and topic time: the case of Mano (Mande). Berkeley.

Lafage, Susanne. 2002. Le lexique fraçais de Côte d'Ivoire (appropriation et créativité). Le français en Afrique. Revue du Réseau des Observatoires du Français Contemporain en Afrique 16-17. http:// www.unice.fr/bcl/ofcaf/16/16.html.

Nedjalkov, Vladimir (ed.). 1988. Typology of resultative constructions. Translated from the original Russian edition (1983). John Benjamins. (Typological Studies in Language 12).

Vydrin, Valentin. 2013. Goo : présentation d'une langue. Mandenkan 50. 171-194.

Vydrin, Valentin. 2014. Goo, neizvestnyj jazyk mande (гоО, НЕИзВЕСтныЙ ЯзЫк МАНДЕ) [Goo, an unknown Mande language]. In Alexander Zheltov (ed.), Antropologija i lingvistika. Materialy peterburgskikh ekspedicij v Afriku (АНТРОПОЛОГИЯ И ЛИНГВИСТИКА. МАТЕРИАЛЫ ПЕТЕРБУРГСКИХ ЭКСПЕДИЦИЙ В АФРИКУ) [Anthropology and linguistics. Materials of field trips to Africa from St. Petersburg], 67-127. St. Petersburg: Muzej antropologii i etnografii RAN (Kunstkamera). 


\section{NOTES}

1. Distance temporelle supérieure à celle entre le kla-dan et le dan-blo, ou entre le gouro et le yaouré qui sont normalement vues comme des langues séparées.

2. Sauf qu'en 2017, une chanson en goo a été diffusée par une station de radio locale.

3. Fotou : un plat national ivoirien, une pâte de consistance élastique, à base de banane, d'igname, de taro, ou de manioc. Présenté sous forme de boule (Lafage 2002).

4. Cf. (Fedotov 2012) où la pertinence de l'attitude de locuteur envers la distance temporelle est analysée par rapport au choix de la construction du futur immédiat.

5. Le commentaire de l'assistant: » On prie Dieu qu'il ne vienne pas ».

6. Les distributions présentées dans les tableaux $6 \mathrm{a}$ et $6 \mathrm{~b}$ sont statistiquement significatives. Les données des tableaux $6 a$ ont été vérifiées à l'aide d'un test statistique chi-carré, ce qui permet de postuler la présence d'une corrélation entre les paramètres testés en comparant la distribution observée avec celle attendue. Le résultat du test est la valeur de p qui désigne la probabilité que la différence entre la distribution observée et la distribution attendue puisse apparaitre au hasard, c'est-à-dire que, plus la valeur de $\mathrm{p}$ est importante, plus la distribution est importante. Dans ce cas, $p<0,0001$, ce qui permet de postuler la présence d'une corrélation entre les paramètres vérifiés.

7. Les données du Tableau $6 \mathrm{~b}$ ont été vérifiées à l'aide du test exact de Fisher, qui analyse la répartition observée. Le test du chi-carré n'a pas été utilisé, car il est inapplicable pour les échantillons où les données de distribution prévues au moins dans une cellule sont inférieures à 5. Quant au test de Fisher, il peut être appliqué à pratiquement n'importe quel échantillon. Selon le test, la probabilité d'une disribution identique dans les données sous l'hypothèse nulle est assez faible: $p<0,05$, ce qui nous permet également de postuler la présence d'une corrélation entre les paramètres vérifiés.

\section{ABSTRACTS}

The article describes the verbal morphology of Goo (Southern Mande). Goo is spoken in the western part of Côte d'Ivoire. The number of speakers is presumably 7-8 thousands. The study was conducted during two field trips in Côte d'Ivoire. In Goo, verbal grammatical meanings are expressed both analitically and synthetically. Analitical means are copulas and predicative markers, inflection is represented by suffixation and tonal alternations. Aspectual meanings predominate with respect to temporal ones. Goo has verbal constructions for habitual, progressive, perfect, resultative, aorist, future, prospective, imperative, subjunctive, continuous, presumptive and two immediate constructions. Negative constructions are less numerous, often a negative construction corresponds to more than one affirmative.

L'article décrit la morphologie verbale de la langue goo (mandé-sud). La langue goo est parlée à l'Ouest de Côte d'Ivoire par sept à huit mille locuteurs. Les langues les plus proches sont le toura et le dan. L'étude est basée sur les donnés recueillies au cours de deux missions de terrain en Côte d'Ivoire. Dans le goo, essentiellement, les valeurs grammaticales verbales sont exprimées par les éléments pronominaux. Les formes verbales subissent les modifications tonales et peuvent ajouter des suffixes en fonction des costructions où ils sont employées. La plupart des constructions expriment des valeurs aspectuelles, plutôt que temporelles. On distingue les 
constructions de l'habituel, du progressif, du parfait, du résultatif, de l'aoriste, du futur, du prospectif, du subjonctif, de l'impératif, du continuatif, du présumptif et les constructions immédiates. Les constructions négatives sont moins nombreuses, souvent une construction négative correspond à plusieurs affirmatives.

В СТАТЬЕ ОПИСЫВАЕТСЯ ГЛАГОЛЬНАЯ МОРФОЛОГИЯ ЯЗЫКА ГОО (ЮЖНЫЕ МАНДЕ). НА ГОО ГОВОРЯТ 7-8 ТЫСЯЧ ЧЕЛОВЕК НА ЗАПАДЕ КОТ-Д'ИВУАРА. НАИБОЛЕЕ БЛИЗКИЕ К НЕМУ ЯЗЫКИ ЭТО ТУРА И ДАН. МАТЕРИАЛЫ ДЛЯ ДАННОГО ИССЛЕДОВАНИЯ БЫЛИ СОБРАНЫ В ХОДЕ ДВУХ ЭКСПЕДИЦИЙ В КОТ Д'ИВУАР. ГЛАГОЛЬНЫЕ ЗНАЧЕНИЯ В ГОО МОГУТ БЫТЬ ВЫРАЖЕНЫ КАК АНАЛИТИЧЕСКИ, ТАК И СИНТЕТИЧЕСКИ. К АНАЛИТИЧЕСКИМ СПОСОБАМ ОТНОСЯТСЯ ПРОНОМИНАЛЬНЫЕ ЭЛЕМЕНТЫ, КОПУЛЫ И ПРЕДИКАТИВНЫЕ ПОКАЗАТЕЛИ. К СИНТЕТИЧЕСКИМ ИЗМЕНЕНИЯ ТОНАЛЬНОГО КОНТУРА И СУФФИКСАЦИЯ. БОЛЬШИНСТВО КОНСТРУКЦИЙ ВЫРАЖАЮТ ВИДОВЫЕ, А НЕ ВРЕМЕННЫЕ ЗНАЧЕНИЯ. В ГОО ВЫДЕЛЯЮТСЯ СЛЕДУЮЩИЕ ГЛАГОЛЬНЫЕ КОНСТРУКЦИИ: ХАБИТУАЛИС, ПРОГРЕССИВ, ПЕРФЕКТ, РЕЗУЛЬТАТИВ, АОРИСТ, БУДУЩЕЕ, ПРОСПЕКТИВ, КОНЪЮНКТИВ, ИМПЕРАТИВ, КОНТИНУАТИВ, ПРЕЗУМПТИВ И ИММЕДИАТНЫЕ КОНСТРУКЦИИ. ОТРИЦАТЕЛЬНЫЕ КОНСТРУКЦИИ МЕНЕЕ МНОГОЧИСЛЕННЫ, ЧАСТО ОДНА ОТРИЦАТЕЛЬНАЯ КОНСТРУКЦИЯ СООТВЕТСТВУЕТ НЕСКОЛЬКИМ УТВЕРДИТЕЛЬНЫМ.

\section{INDEX}

Mots-clés: Goo, Mandé-Sud, morphologie verbale, le système de temps-aspect-modalité motsclesru ГОО, ЮЖНЫЕ МАНДЕ, ГЛАГОЛЬНАЯ МОРФОЛОГИЯ, АСПЕКТО-ТЕМПОРАЛЬНОМОДАЛЬНАЯ СИСТЕМА

Keywords: Goo, South Mande, verbal morphology, TAM system

\section{AUTHOR}

\section{EKATERINA APLONOVA}

National Research University Higher School of Economics, Moscow aplooon@gmail.com 